\title{
Beverages sales following the implementation of the Healthier Vending Policy across Health Service Executive premises in the Republic of Ireland
}

\author{
S. Bel-Serrat, I. Stanley ${ }^{1}$, A. Lawless ${ }^{2}$, S. O’Brien ${ }^{2}$, C.C. Kelleher ${ }^{1}$ and C.M. Murrin ${ }^{1}$ \\ ${ }^{1}$ National Nutrition Surveillance Centre, School of Public Health, Physiotherapy and Sports Science, University \\ College Dublin, Dublin, Ireland and ${ }^{2}$ Healthy Eating \& Active Living Programme, Health Service Executive, Dublin, \\ Ireland.
}

The Healthy Ireland Survey 2017 showed that $39 \%$ and $23 \%$ of the population in the Republic of Ireland are overweight and obese, respectively ${ }^{(1)}$. Besides, $16 \%$ drink sugar sweetened beverages on a daily basis and $38 \%$ at least once a week ${ }^{(1)}$. Reducing sugar sweetened beverages consumption may help maintain a healthy body weight. Vending machines typically offer energy-dense, nutrient-poor foods and beverages, and few healthy options, and contribute to the obesogenic environment with constant exposure and access to unhealthy foods and beverages ${ }^{(2)}$. Increasing availability of healthier products may be sufficient to influence consumer choices ${ }^{(2)}$.

In 2015, the Health Service Executive (HSE) implemented the Healthier Vending Policy to promote healthier food environments by increasing healthier choices and reducing unhealthy food choices in vending machines located in the HSE premises across the Republic of Ireland. The Policy applies to cold soft drinks, confectionery and snacks in all the vending machines in the HSE premises $^{(3)}$. All products stocked in vending machines under the Policy are required to meet the classification of Better Choice (BC) or Other Choice (OC) products according to specific criteria on energy content per packet and nutrient content (total fat, saturated fat, sugar, and sodium/salt) per $100 \mathrm{~g}^{(3)}$. Furthermore, vending machines are required to stock $\mathrm{BC}$ and $\mathrm{OC}$ products to a ratio of 60:40.

We aimed to examine the compliance of beverages vending machines with the 60:40 ratio and the proportion of $\mathrm{BC}$ versus $\mathrm{OC}$ beverages sales following the implementation of the policy. A single-arm policy evaluation was conducted using 2016 annual beverages sales provided by the two companies in the framework. Water, diet soft drinks, and fruit juices were classified as BC beverages. Water was further sub-categorised into still, sparkling and flavoured water. The proportion of total beverage sales was calculated. The proportion of $\mathrm{BC}$ and $\mathrm{OC}$ beverages stocked in vending machines was calculated through machines planograms. Compliance with the 60:40 ratio was examined separately for each company.

A total of 295 vending machines across 89 HSE sites were included in the analyses. All beverages machines met the 60:40 ratio of BC:OC beverages to a ratio of $80: 20$ for company 1 and $67: 33$ for company 2 . Sales of BC beverages accounted for $65 \%$ of total beverage unit sales. Diet soft drinks and total water sales accounted for $54 \%$ and $37 \%$ of BC beverage sales, respectively. Fruit juices only represented $9 \%$ of $\mathrm{BC}$ beverages sales. Focusing on water types, still water accounted for $28 \%$, flavoured water for $6 \%$, and sparkling water for $3 \%$ of total BC beverage sales. Sales of 'Other Choice' beverages (35\%) were entirely attributable to soft drink sales.

In conclusion, sales of $\mathrm{BC}$ beverages were higher as compared with $\mathrm{OC}$ beverages sales. Diet soft drinks were the most purchased beverages followed by still water. Soft drinks represented one third of total beverages sales. The high availability of BC beverages in vending machines may have had an impact on healthier consumer beverage choices. Overall, the implementation of the vending machines policy seemed to promote a healthier environment in terms of beverages purchase across the HSE premises. This was the first evaluation of the policy, therefore, further follow-up analyses are needed to observe trends in BC and OC beverages sales to monitor the progress of the implementation of the policy.

1. Healthy Ireland Survey (2017) Department of Health, Dublin.

2. Hua SV, Kimmel L, Van Emmenes M et al. (2017) J Acad Nutr Diet 117, 1057-1065.

3. Health Service Executive - Healthier Vending Policy (2015) Health Service Executive, Dublin. 